\section{Posicionamiento de los investigadores en el espacio social del Instituto de Investigaciones sobre la Universidad y la Educación, México}

\author{
Positioning of researchers in the social space of the Instituto de Investigaciones sobre la \\ Universidad y la Educación, Mexico
}

Sara Bravo Villanueva; Luis Arturo Guerrero Azpeitia
INFORMACIÓN:

http://doi.org/10.46652/runas.v2i4.50 ISSN $2737-6230$

Vol. 2, No. 4, 2021. e21050

Quito, Ecuador

Enviado: septiembre 26, 2021 Aceptado: noviembre 27, 2021 Publicado: diciembre 11, 2021 Sección Dossier | Peer Reviewed Publicación continua que da pauta a pensar, percibir, decidir y actuar de una manera particular. Metodológicamente se recuperaron técnicas que potencializaron la relación entre las variables analíticas y las categorías interpretativas, posibilitando de esta manera la articulación entre las condiciones objetivas y la producción de subjetividad. De los principales resultados, se destaca la construcción analítica de clases sociales en el espacio académico del IISUE, a saber: consolidados, principiantes y heréticos; en adición, dicha construcción permitió relacionar las disposiciones de las y los investigadores que regulan las prácticas de diferenciación y clasificación de la comunidad científica. A manera de conclusión, se identificó que el volumen y estructura de capital de los investigadores configura y reconfigura el campo académico y es a través de sus prácticas sociales que tienden a reproducir los mecanismos de poder y desigualdad propios del campo bajo análisis.

Palabras clave: espacio social; campo científico; investigadores educativos; capitales; construcción de clases sociales

\section{ABSTRACT}

The institutionalization of educational research in Mexico has led to a professionalized and professionalizing practice, with a peculiar recognition and status, in this aspect, the objective was to identify the influence of material and symbolic conditions, as well as the appropriation of capital that regulates the production and reproduction of the academic practices of the researchers of the Research Institute on the University and Education, of the National Autonomous University of Mexico (UNAM-IISUE). For this purpose, Bourdieu's Theory of the Economics of Social Practices was recovered, which establishes that social relations do not occur spontaneously or casually but are conditioned by a social environment that gives guidelines to think, perceive, decide and act in a particular way. Methodologically, techniques were recovered that potentiate the relationship between analytical variables and interpretative categories, thus enabling the articulation between objective conditions and the production of subjectivity. Of the main results, the analytical construction of social classes in the academic space of the IISUE stands out, namely: consolidated, beginners and heretical; in addition, this construction allowed to relate the provisions of the researchers that regulate the practices of differentiation and classification of the scientific community. By way of conclusion, it was identified that the volume and capital structure of researchers configures and reconfigures the academic field and it is through their social practices that they tend to reproduce the mechanisms of power and inequality of the field under analysis.

Keywords: social space; scientific field; educational researchers; capitals; construction of social classes

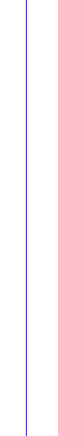

(

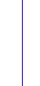

\section{AUT}

UTORES:

Sara Bravo Villanueva Universidad Nacional Autónoma de México - México

sarabrav01619@gmail.com

Luis Arturo Guerrero Azpeitia Universidad Politécnica Metropolitana de Hidalgo - México Iguerrero@upmh.edu.mx

\section{CONFLICTO DE INTERESES}

Los autores declaran que no existe conflicto de interés posible.

FINANCIAMIENTO

No existió asistencia financiera de partes externas al presente artículo. NOTA

El artículo es producto de tesis de doctorado en pedagogía, actualmente en desarrollo.

PUBLISHER

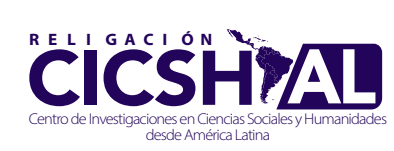




\section{Introducción}

La investigación educativa en México ha atravesado por un importante periodo de institucionalización que le ha permitido convertirse en una práctica profesionalizada y profesionalizante, con reconocimiento y estatus dentro de la comunidad académica y de la sociedad en general, (Gutiérrez, 1998; Cabrera, 2013); el Instituto de Investigaciones sobre la Universidad y la Educación (IISUE), de la Universidad Nacional Autónoma de México (UNAM) es un espacio en donde se ha contribuido al desarrollo de la educación como ciencia y la investigación se ha institucionalizado, dado que tiene como función sustantiva la investigación educativa y de la universidad, se ha preocupado por crear las condiciones institucionales necesarias para su desarrollo, tales como: la contratación de académicos de tiempo completo con nombramiento de investigadores; su desarrollo y producción académica le han constituido en un referente nacional y regional dentro del campo disciplinar por el prestigio que sus investigadores han logrado (UNAM/CESU, 2006).

El Instituto tiene por actividad sustantiva investigar sobre la problemática de la universidad y la educación, así como albergar, custodiar y proyectar el Archivo Histórico de la UNAM. Sus líneas de investigación se han ampliado en una diversidad de aspectos temáticos, constituyéndose en una entidad de investigación y desarrollo que cubre distintos campos de construcción teórica y aplicada, relacionada con la complejidad de los fenómenos educativos y de la universidad, y que abarca distintas disciplinas, metodologías, enfoques y campos de la educación (UNAM/IISUE, 2018).

Esta investigación tuvo como propósito identificar las condiciones materiales, simbólicas y apropiación de capitales que permiten a sus investigadores el desarrollo de una carrera académica en el IISUE, ya que la apropiación de recursos por parte de sus investigadores contribuye en la construcción y estructuración del espacio y de las relaciones que los vincula; este acercamiento fue desarrollado analíticamente con el interés de contribuir en el conocimiento del espacio social como lugar de producción y reproducción de prácticas científicas para la investigación educativa, las cuales se han modificado al paso de su historia, constituyéndose en lugares de iniciación, formación, confluencia y encuentro de los hoy investigadores del IISUE.

EI IISUE es producto de varios acuerdos académicos que dieron pie a procesos de transformación de unidades académicas y centros de investigación que le antecedieron; en 1976 se conforma el Centro de Estudios sobre la Universidad (CESU) incorporando al Archivo Histórico que se había fundado en 1964; en 1977 se creó el Centro de Investigaciones y Servicios Educativos (CISE) a partir de la fusión del Centro de Didáctica (1969) y la Comisión de Nuevos Métodos de Enseñanza (1969); en 1985 se integran al CESU el grupo de investigadores del Departamento de Estudios Educativos (1979) que estaban adscritos a la Coordinación de Humanidades; en 1997 se suman al CESU los académicos del CISE que traen consigo la publicación Perfiles Educativos, revista que representa uno de los espacios más importantes para la difusión de los nuevos conocimientos generados a partir de las investigaciones realizadas en nuestro país, América Latina y otros países de habla hispana, principalmente, y que además en el año 2000, fue una de las primeras revistas educativas reconocidas por el Índice de Revistas Mexicanas de Investigación Científica 
y Tecnológica (IRMICyT) del Consejo Nacional de Ciencia y Tecnología (CONACYT), avalando de esta forma la alta calidad académica de la revista; y finalmente en 2006, el reconocimiento de su consolidación académica y sus aportaciones a la investigación educativa le permitieron al CESU transitar de centro de investigación a conformarse como Instituto de Investigaciones sobre la Universidad y la Educación, ahora con mejores condiciones materiales y sociales para el desarrollo de la práctica de la investigación educativa (UNAM/CISE, 1983; UNAM/CESU, 1997; UNAM/CESU, 2000; UNAM/CESU, 2006; Chehaibar y Paniagua, 2009).

\section{Metodología}

La elección de un método específico para la realización de la investigación, es sin duda un aspecto estratégico y demanda, a la vez, precisar los referentes conceptuales que se utilizarán para estudiar esa parte de la realidad social que nos propusimos comprender, determinando una lógica de aproximación empírica, donde el qué y el cómo indagar están prefigurados por ciertos principios metodológicos de la perspectiva conceptual elegida.

Bourdieu y Wacquant precisan que existe una falsa separación entre teoría, metodología y trabajo empírico que la selección de las “(...) técnicas más ‘empíricas’ son inseparables de las elecciones más 'teóricas' de construcción del objeto. Siempre es en función de cierta construcción del objeto que se impone tal método de muestreo, determinada técnica de acopio o de análisis de datos, etc." (Bourdieu y Wacquant, 1995, p. 167); por consiguiente, toda decisión dentro del marco de la investigación trae implícita una perspectiva epistemológica particular y específicamente esta indagación se desarrolló adoptando la propuesta teórico metodológica de Pierre Bourdieu.

Para Bourdieu (2011) las relaciones sociales no se dan de manera espontánea ni casual, sino que están condicionadas por un entorno social que da pauta a pensar, percibir, decidir y actuar de una y no de otra manera. Todas las estructuras sociales que conforman las relaciones de un espacio social son mecanismos de transmisión, sostenimiento y estimulación de ciertas prácticas que se desarrollan como una adaptación a las condiciones económicas, sociales y culturales comunes al grupo de personas que pertenecen a dicha estructura; por ello, un punto de partida del análisis de lo social lo constituye el abocarnos a caracterizar las estructuras sociales externas, lo objetivo, lo social hecho cosa, esas relaciones sociales que no se eligen, que existen independientemente de la voluntad y conciencia de los sujetos, y que fungen como condicionamientos, de acuerdo con Bourdieu y sus colegas "no hay conducta, actitud o ideología alguna que pueda ser descrita, comprendida o explicada objetivamente al margen de toda referencia a la situación existencial" (Bourdieu et al., 1963, p. 258).

Bourdieu considera que la construcción empírica de las relaciones sociales objetivas pasa por responder qué hace diferentes a los sujetos de un grupo social, y lo que los hace diferentes son sus propiedades, esos recursos materiales y simbólicos con los cuales va generando su existencia y posicionándolos en el espacio social, conformando desigualdades sociales que se constituyen en principios de diferenciación, de distinción (Bourdieu, 1990, 1997, 2011, 2014). Una forma de categorizar las desigualdades sociales más significativas que empíricamente se presentan entre los sujetos es diferenciarlos por clases sociales y las relaciones que se establecen entre las clases sociales son relaciones de dominación. 
Quienes mejor se han apropiado de los recursos materiales y simbólicos, capitales para Bourdieu, característicos del grupo social son quienes mejor conocen y han participado en la definición y establecimiento de las clasificaciones, y es a través de las instituciones del Estado que se imponen las leyes, normas, reglas, intereses, ideas, creencias, derechos y jerarquías que regulan las relaciones entre los sujetos, constituyen el campo de poder y tienen la autorización, conocimiento y reconocimiento para definir la información aceptable, verdadera y legítima. El Estado como principio organizador y clasificador, a través de sus instituciones, impone un orden social (Bourdieu, 2014, 2001a).

En México han sido la Academia Mexicana de la Ciencia (AMC), la Asociación Nacional de Universidades e Instituciones de Educación Superior (ANUIES), el CONACyT, el Sistema Nacional de Investigadores (SNI) y la UNAM, como instituciones de Estado, quienes han normado la ciencia y constituyen el campo de poder en donde se ubican los científicos destacados, de prestigio nacional e internacional que junto a las autoridades que presiden a dichas instituciones han hecho pre-visible, concebible y creíble un modelo especifico de investigación científica para nuestro país, creando la representación y voluntad para hacerla posible, han marcado la pauta del quehacer y reconocimiento científico. Al enunciar el quehacer de la investigación, este grupo dominante ha ido creando enunciados performativos (Bourdieu, 2001b) que ordenan y deciden cuales son las prácticas científicas más adecuadas y pertinentes de la investigación en México.

Para el caso de este estudio resultó imprescindible la identificación de los principios de clasificación, diferenciación, inclusión, sanción o exclusión de las normas de la investigación científica, por ser los criterios que estructuran el espacio social y ubican a los investigadores en una posición determinada, de acuerdo a la apropiación de los recursos materiales y simbólicos socialmente disponibles. De esta manera, la pregunta que guio esta indagación fue: ¿cuáles son los recursos materiales y simbólicos de los investigadores del IISUE, mediante los cuales desarrollan la práctica de la investigación educativa?

Algunos principios que articulan las diferencias sociales y culturales en el espacio de la investigación educativa que se desarrolla en el IISUE, están inscritos en la norma, están estipulados formal y jurídicamente en el Estatuto del Personal Académico (EPA) de la UNAM, otorgándoles derechos y obligaciones a sus investigadores, mientras que otros principios de diferenciación están determinados por las tradiciones en la investigación educativa, estableciendo el tipo de relaciones sociales dentro del IISUE de acuerdo a las condiciones y posiciones sociales de los investigadores. En principio el EPA (UNAM, s/f) señala que los investigadores serán siempre de carrera y con dedicación de tiempo completo; para su ingreso deberán ser seleccionados por concurso de oposición abierto para ingreso y cerrado para promoción; transitar por la escala jerárquica conformada por categorías y niveles (Asociado y Titular A, B y C, interino o definitivo); poseer bienes simbólicos como lo son el grado académico y el reconocimiento gremial.

En consecuencia, buscar las evidencias empíricas de los recursos que poseen los investigadores implicó partir de aquellos aspectos normativos y prácticos que significaran diferencias y clasificaciones de los investigadores, por la posesión de propiedades materiales y simbólicas tales como: 
- Nombramiento académico: porque significa un lugar en la escala jerárquica de las contrataciones.

- Grado académico: reconocimiento institucional de la posesión de conocimiento especializado.

- Nivel SNI: distinción de prestigio como Investigador Nacional con escala jerárquica.

- Nivel PRIDE: reconocimiento universitario por desempeño académico sobresaliente para académicos de tiempo completo con escala jerárquica.

- Producción académica: la investigación cobra sentido a partir de la publicación de los resultados de la misma, además conforma la relación que los investigadores establecen con sus pares para debatir sus planteamientos, les da visibilidad en el campo científico y en consecuencia prestigio académico en virtud de la difusión de sus ideas. Para esta variable se consideraron la publicación de artículos, libros de autor, capítulos de libros y libros coordinados.

- Edad: expresa diferencias vinculadas a las etapas del ciclo de vida y a la configuración de prácticas específicas de investigación de acuerdo a la época de inserción en el trabajo de investigador.

- Género: característica cultural que diferencia a hombres y mujeres y puede facilitar o dificultar el desarrollo de trayectorias académicas.

- Antigüedad académica: trayectoria en el tiempo de dedicación a la investigación.

Un primer momento del análisis de la información, recopilada a través de una matriz de frecuencias (base de datos organizada en una hoja de cálculo de Excel), sobre las principales características de los investigadores del IISUE, fue relacionar algunas variables consideradas pertinentes, es decir, realizar un cruce de dos variables para presentar de manera gráfica una imagen de quienes son los investigadores del Instituto.

Un segundo momento del análisis estadístico para dar cuenta de las condiciones materiales de existencia de los investigadores del IISUE fue mediante el Análisis de Correspondencias Múltiples (ACM); el ACM permite presentar de una manera gráfica la estructura del campo, la cual hace referencia a la apropiación desigual de recursos (capitales) que al mismo tiempo nos habla de sus prácticas de los investigadores del IISUE.

El desarrollo de métodos de análisis multivariado mediante el análisis de correspondencias múltiples tuvo su origen en la "escuela francesa de análisis de datos" con base en la obra de Jean-Paul Benzécri (Baranger, 2009); la técnica de ACM fue difundida ampliamente por Bourdieu y sus colaboradores para presentar los resultados de sus investigaciones empíricas y la podemos apreciar en sus obras tales como: La distinción (1998), Homo Academicus (2008), Los herederos (Bourdieu y Passeron, 2008), por mencionar solo algunas.

EI ACM es una técnica de análisis de datos multivariable destinada a mostrar la relación de variables cualitativas, tratadas como nominales u ordinales, y que expresan los principales factores de diferenciación, construyendo clases a partir de la ubicación de los sujetos según la relación con las variables, buscando analizar, describir y representar gráficamente las correspondencias (asociaciones) de información contenidas en un diagrama de distribución. 
En la técnica de ACM las variables pueden jugar dos papeles: activo e ilustrativo. Las variables activas son las que intervienen en la generación de las clases, mientras que las variables ilustrativas no contribuyen en la construcción del espacio, pero si son proyectadas en el diagrama porque tienen el objetivo de mostrar la relación que hay con respecto a las otras variables (Moscoloni, 2011).

Una variable es una característica que toma distintos valores para cada uno de los sujetos, en este caso la población en estudio son los 67 investigadores adscritos al IISUE, en la matriz de frecuencias se registraron sus datos, los cuales constituyen propiedades de distinción; para el ACM tomamos variables nominales u ordinales que representan valores categóricos que surgen de una operación clasificadora, posteriormente se les asignó una codificación, es decir, la determinación de valores (pueden o no ser numéricos) representativos de las variaciones que pueda presentar, para posteriormente establecer una escala de medición, o sea asignarle un valor numérico que jerarquice la codificación, porque es la técnica que nos permite habilitar las variables para ser procesadas.

Esquema 1. Estructura de base de datos

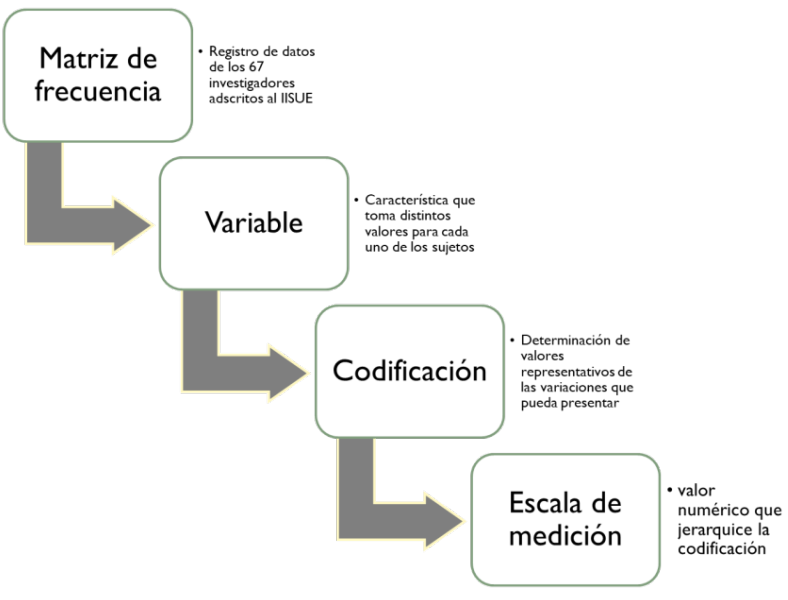

Fuente: Elaboración propia.

A las variables seleccionadas se les asignó una codificación y una escala específica para favorecer el procesamiento de los datos a través del SPSS, quedando de la siguiente manera:

Contratación: de acuerdo al EPA puede ser interino, definitivo o por artículo 51 (temporal y por obra determinada).

Tabla 1. Contratación de investigadores

\begin{tabular}{|l|r|}
\hline \multicolumn{1}{|c|}{ Contratación } & \multicolumn{1}{|c|}{ Escala } \\
\hline Art. 51 & 1 \\
\hline Interino & 2 \\
\hline Definitivo & 3 \\
\hline
\end{tabular}

Fuente: Elaboración propia.

Nombramiento UNAM: de acuerdo a las categorías de contratación estipuladas en el EPA. 
Tabla 2. Nombramiento de investigadores

\begin{tabular}{|l|r|}
\hline \multicolumn{1}{|c|}{ Nombramiento UNAM } & Escala \\
\hline Asociado A & 2 \\
\hline Asociado B & 2 \\
\hline Asociado C & 3 \\
\hline Titular A & 4 \\
\hline Titular B & 5 \\
\hline Titular C & 6 \\
\hline Emérito & 7 \\
\hline
\end{tabular}

Fuente: Elaboración propia.

Antigüedad: se tomó como referencia el año de 1979 por ser el registro más antiguo que presenta uno de los investigadores del IISUE hasta 2019, año en que se elaboró la matriz para el procesamiento de datos estadísticos, esta variable se dividió por periodos, los cuales coinciden también con ciertas etapas de vida de las entidades académicas que antecedieron al IISUE y donde se fue desarrollando la práctica de la investigación educativa en la UNAM, además se le determinó una codificación: Consolidado.- para el grupo de investigadores de mayor antigüedad en la práctica de la investigación, tienen más de veinte años, y que ingresaron entre 1979 y 1996; al segundo grupo lo denominamos Experimentado.- son investigadores que ingresaron entre 1997-2010; finalmente está el grupo Principiante.- son investigadores que comienzan a realizar la actividad de la investigación de manera profesional y se incorporaron entre 2011-2019. A estos grupos se les definió una escala de medición que los jerarquiza, quedando de la siguiente manera:

Tabla 3. Antigüedad de investigadores en el IISUE

\begin{tabular}{|l|r|r|}
\hline $\begin{array}{c}\text { Antigüedad } \\
\text { (año de ingreso) }\end{array}$ & Codificación & \multicolumn{1}{c|}{ Escala } \\
\hline $2011-2019$ & PRINCIPIANTE & 1 \\
\hline $1997-2010$ & EXPERIMENTADO & 2 \\
\hline $1979-1996$ & CONSOLIDADO & 3 \\
\hline
\end{tabular}

Fuente: Elaboración propia.

Grado académico: denota el reconocimiento institucional de la posesión de conocimiento especializado.

Tabla 4. Grado académico de investigadores

\begin{tabular}{|l|r|}
\hline \multicolumn{1}{|c|}{ Grado académico } & Escala \\
\hline Maestro & 1 \\
\hline Doctor & 2 \\
\hline
\end{tabular}

Fuente: Elaboración propia.

Publicaciones: esta variable se construyó a partir de la información disponible en el portal de HUMANINDEX de la UNAM y fueron considerados los artículos, capítulos de libros, libros de autor y libros coordinados, por ser los productos más relevantes de la investigación y ser además el principal indicador para la evaluación los investigadores en los programas de estímulos, se estableció un rango, una codificación y una escala jerárquica. 
Tabla 5. Publicaciones por rango

\begin{tabular}{|l|r|r|}
\hline \multicolumn{1}{|c|}{ Publicaciones } & \multicolumn{1}{|c|}{ Codificación } & \multicolumn{1}{c|}{ Escala } \\
\hline $0-10$ & MUY BAJO & 1 \\
\hline $11-50$ & BAJO & 2 \\
\hline $51-100$ & MEDIO & 3 \\
\hline $101-150$ & ALTO & 4 \\
\hline MAS DE 150 & MUY ALTO & 5 \\
\hline
\end{tabular}

Fuente: Elaboración propia.

PRIDE: reconocimiento universitario por desempeño académico sobresaliente para académicos de tiempo completo, a esta variable se le asignó una escala de acuerdo a la jerarquización que el mismo programa especifica.

Tabla 6. Niveles del Programa de Primas al Desempeño del Personal Académico de Tiempo Completo (PRIDE)

\begin{tabular}{|l|r|}
\hline \multicolumn{1}{|c|}{ PRIDE } & \multicolumn{1}{|c|}{ Escala } \\
\hline NADA & 1 \\
\hline A & 2 \\
\hline B & 3 \\
\hline C & 4 \\
\hline D & 5 \\
\hline
\end{tabular}

Fuente: Elaboración propia.

SNI: la distinción como Investigador Nacional establece una escala jerárquica misma que se recuperó para el ACM.

Tabla 7. Niveles del Sistema Nacional de Investigadores

\begin{tabular}{|l|r|}
\hline \multicolumn{1}{|c|}{ SNI } & \multicolumn{1}{|c|}{ Escala } \\
\hline S/SNI & 1 \\
\hline Candidato & 2 \\
\hline 1 & 3 \\
\hline II & 4 \\
\hline III & 5 \\
\hline SNI/EMÉRITO & 6 \\
\hline
\end{tabular}

Fuente: Elaboración propia.

Grupo etario: esta fue tomada como variable ilustrativa y construida tomando como referencia la edad del investigador más joven y haciendo rangos hasta llegar a más de 70 años, agregándoles una codificación y una escala.

Tabla 8. Grupo etario

\begin{tabular}{|c|c|c|}
\hline Grupo etario (edad) & Codificación & Escala \\
\hline $35-50$ & JOVEN & 1 \\
\hline $51-70$ & MADURO & 2 \\
\hline más de 70 & MAYOR & 3 \\
\hline
\end{tabular}

Fuente: Elaboración propia. 
Género: esta variable también fue tomada como ilustrativa a fin de conocer si existen diferencias entre hombres y mujeres que puedan facilitar o dificultar el desarrollo de trayectorias académicas.

Tabla 9. Sexo de investigadores

\begin{tabular}{|l|r|r|}
\hline \multicolumn{1}{|c|}{ Sexo } & Codificación & \multicolumn{1}{|c|}{ Escala } \\
\hline Femenino & F & 1 \\
\hline Masculino & $\mathrm{M}$ & 2 \\
\hline
\end{tabular}

Fuente: Elaboración propia.

El ACM permite ver el tipo de asociación existente entre variables, mostrándonos todos los cruzamientos posibles entre las mismas y está centrado en la configuración de diagramas que muestran la posición social de los sujetos de acuerdo a la posesión de capitales que ha movilizado para participar en la disputa por pertenecer al campo.

Cabe señalar que, todas las gráficas y diagramas de este documento son elaboraciones propias realizadas a partir de información oficial disponible en las páginas electrónicas de: UNAM-IISUE; Base de Datos Bibliográfica de Humanidades y Ciencias Sociales de la UNAM (HUMANINDEX); Dirección General de Asuntos del Personal Académico de la UNAM (DGAPA); Sistema Integral de Administración Escolar del Posgrado de la UNAM (SAEP); y Sistema Nacional de Investigadores (SNI) del CONACYT.

\section{Resultados}

El primer ejercicio de análisis, el cruce de variables, permitió determinar las siguientes características de los investigadores del IISUE: su planta académica está integrada por 67 investigadores, 44 titulares y 23 asociados (Gráfica 1), y su edad promedio es de 58 años (Gráfica 2).

Gráfica 1. Distribución de investigadores por categoría de contratación UNAM, 2019

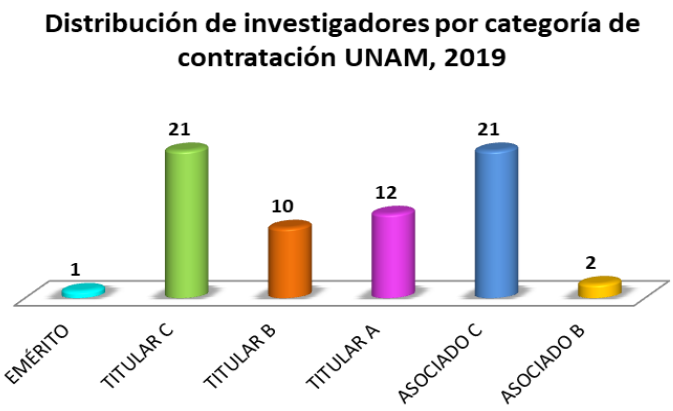

Fuente: Elaboración propia con datos de Instituto de Investigaciones sobre la Universidad y la Educación (IISUE). 
Gráfica 2. Número de investigadores por rango de edad y género.

\section{Número de investigadores por rango de edad y género}

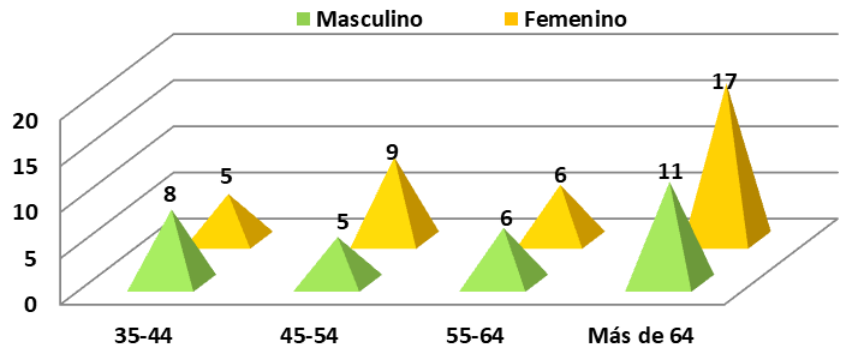

Fuente: Elaboración propia con datos de Instituto de Investigaciones sobre la Universidad y la Educación (IISUE) y el Sistema Integral de Administración Escolar del Posgrado (SIAE-P).

La identificación de los principios de clasificación de las normas de la investigación científica de los programas de evaluación académica, SNI y PRIDE, son los criterios que ubican a los investigadores en una posición determinada en el campo de la investigación científica, de acuerdo al nivel alcanzado en cada uno de los programas de estímulos.

Al revisar el nivel alcanzado en los programas de estímulos al desempeño, de los investigadores del Instituto se determinó que durante el 2019 el 97\% de sus investigadores están incluidos en el Programa de Primas para el Desempeño Académico (PRIDE): $34 \%$ se ubican en el nivel B, $36 \%$ en el C, y $27 \%$ en el nivel D (Gráfica 3).

Gráfica 3. Porcentaje de participación en PRIDE

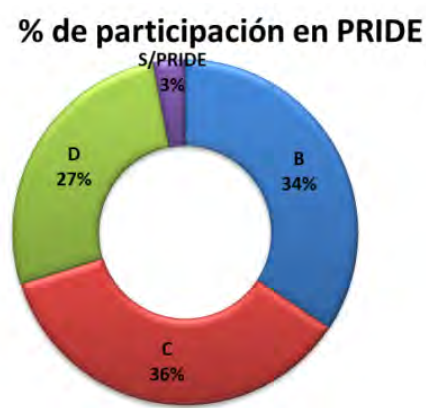

Fuente: Elaboración propia con datos del Instituto de Investigaciones sobre la Universidad y la Educación (IISUE) y de la Dirección General de Asuntos del Personal Académico.

Mientras que el $75 \%$ son parte del Sistema Nacional de Investigadores (SNI), 9\% se ubica en el nivel III, $26 \%$ nivel II, 30\% nivel I y $9 \%$ son candidatos, una cuarta parte de los investigadores no participan en el SNI (Gráfica 4). 
Gráfica 4. Participación de investigadores en el SNI, 2019

Participación de investigadores en el SNI, 2019

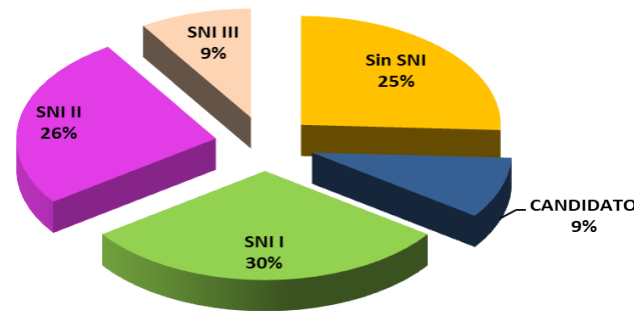

Fuente: Elaboración propia con datos del Instituto de Investigaciones sobre la Universidad y la Educación (IISUE) y del Consejo Nacional de Ciencia y Tecnología.

Para el análisis de la productividad académica se construyeron rangos que representaran niveles de productividad: el primer rango representa la cantidad mínima de publicaciones que va de o a 10 y se le asignó la categoría de nivel de productividad $B A J A$, de 11 a 80 publicaciones nivel MEDIA, de 81 a 150 nivel ALTA y más de 150 MUYALTA.

Al cruzar las variables de rango de publicaciones con género se puede observar que prevalece, tanto en hombres como en mujeres, una productividad MEDIA, que solo 4 investigadores tienen un nivel de producción MUYALTA, 9 investigadores ( 4 hombres y 5 mujeres) productividad ALTA y 16 investigadores tienen menos de 10 publicaciones ubicándose en el nivel BAJA (Gráfica 5).

Gráfica 5. Número de investigadores por género y rango de publicaciones

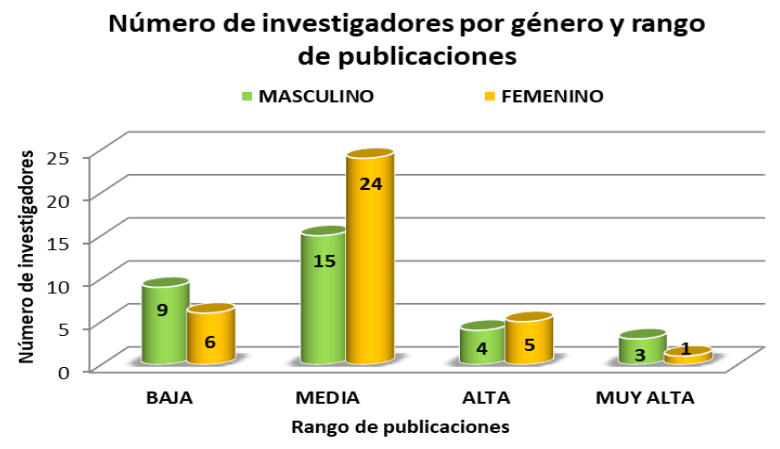

Fuente: Elaboración propia con datos de Instituto de Investigaciones sobre la Universidad y la

Educación (IISUE) y HUMANINDEX.

Cuando se compara el nivel de productividad con el grado académico se puede apreciar la presencia de un pequeño grupo (8 investigadores con grado de maestría) y con niveles de productividad BAJA y MEDIA, mientras que un amplio grupo (32 investigadores) con grado de doctorado se ubican en un nivel de productividad MEDIA y los investigadores con productividad ALTA y MUY ALTA tienen grado de doctores (Gráfica 6). 
Gráfica 6. Número de investigadores por grado académico y rango de publicaciones

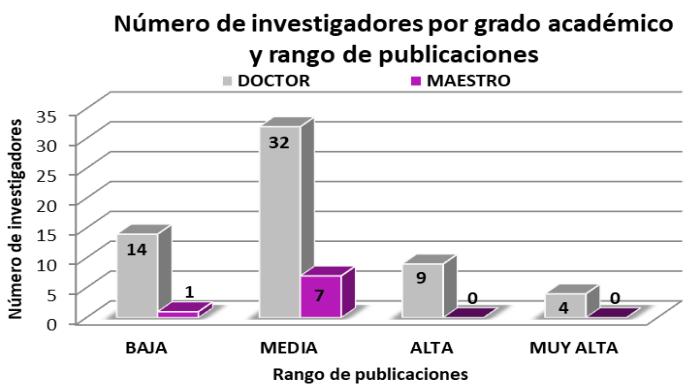

Fuente: Elaboración propia con datos de Instituto de Investigaciones sobre la Universidad y la Educación (IISUE) y HUMANINDEX.

El grado académico es el reconocimiento institucional de la posesión del conocimiento y en la gráfica anterior es evidente que ha habido una preocupación por que la mayoría de los investigadores del Instituto posean el grado máximo de estudios, por ende, la mayoría de sus investigadores son doctores.

El segundo análisis estadístico realizado fue mediante el análisis de correspondencias múltiples. El ACM “es una técnica relacional de análisis de datos cuya filosofía se corresponde exactamente, a mi modo de ver, con aquello que es la realidad del mundo social. Se trata de una técnica que 'piensa' en términos de relación” (Bourdieu y Wacquant, 2005, p. 149). El ACM permite la representación gráfica de la estructura que emerge de la interrelación de las características que portan los sujetos, constituidos como unidades de análisis, posibilitando la visualización de sus similitudes y diferencias (Baranger, 2009).

A través del ACM se puede identificar las relaciones sociales que se dan dentro de un campo, ubican a los sujetos en un lugar del espacio social, ocupando una posición, el conjunto de posiciones y las relaciones entre posiciones nos da la estructura social objetiva, la estructura del campo. Analizar esas estructuras sociales es develar lo que está oculto en las relaciones sociales, es buscar el vínculo que se establece entre los sujetos sociales y el espacio físico, es articular los elementos que operan de manera invisible, buscando las evidencias empíricas de los recursos que poseen y hacen la diferencia, clasificación, vinculación y articulación entre ellos, relaciones que están construidas bajo principios de diferenciación que se constituyen en propiedades actuantes, que otorgan fuerza y poder a quienes las poseen; relaciones sociales que no se ven pero que la investigación social nos permite construir (Bourdieu, 1990 y 2001a).

Con el ACM obtenemos una fotografía del espacio social tomada en un momento determinado del tiempo, la interpretación de los diagramas obtenidos podemos hacerla primero de manera parcial, es decir, analizando el comportamiento de una variable revisando y analizando la cercanía o lejanía entre sus categorías, por ejemplo: Observar la proximidad entre dos puntos de dos categorías de una misma variable, nos indica similitud entre sus perfiles. En el Diagrama 1 está resaltada la variable de Sexo y podemos observar la cercanía que hay entre sus dos categorías, femenino y masculino, lo que nos indica que no existen diferencias significativas determinadas por el sexo del investigador, lo que evidencia que son muy similares los perfiles entre las mujeres y los hombres investigadores del IISUE. 
Diagrama 1. Variable de Sexo

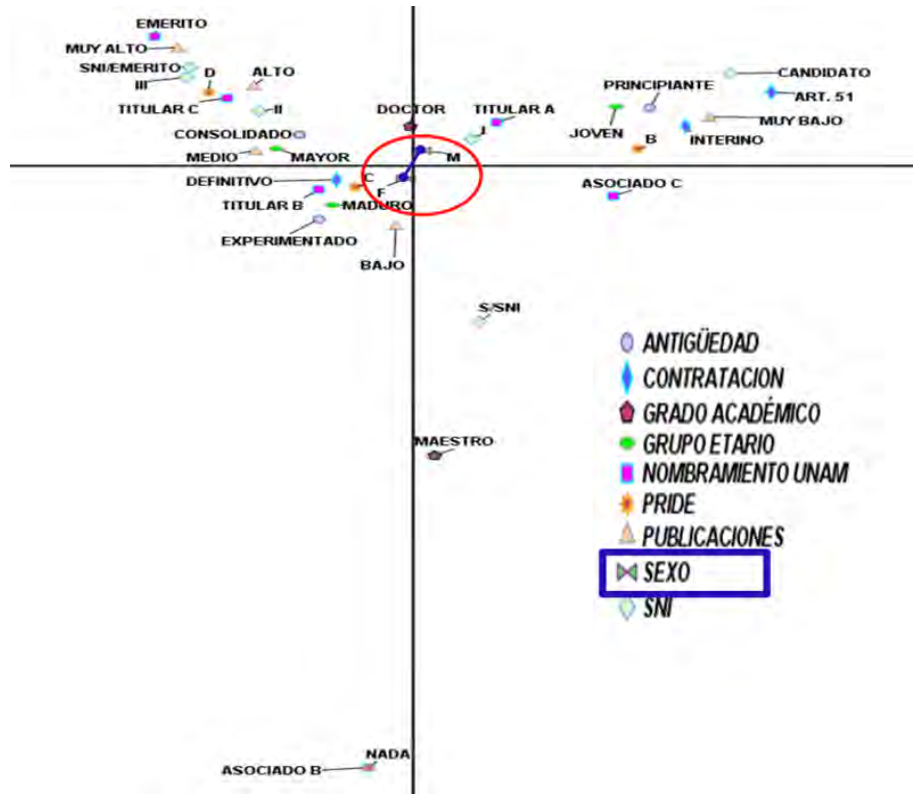

Fuente: Elaboración propia con datos de Instituto de Investigaciones sobre la Universidad y la Educación (IISUE) y HUMANINDEX, CONACYT, DGAPA, SAEP.

La lejanía entre dos puntos de dos categorías de una misma variable indica diferencias entre sus perfiles. En el Diagrama 2 está resaltada la variable Grado académico y la distancia que hay entre ambas categorías da cuenta de diferencias significativas entre las características que las acompañan, en este ejemplo la distancia entre las categorías significa que las características que acompañan a un investigador con grado de maestro son muy diferentes a las de un doctor.

Diagrama 2. Variable Grado académico

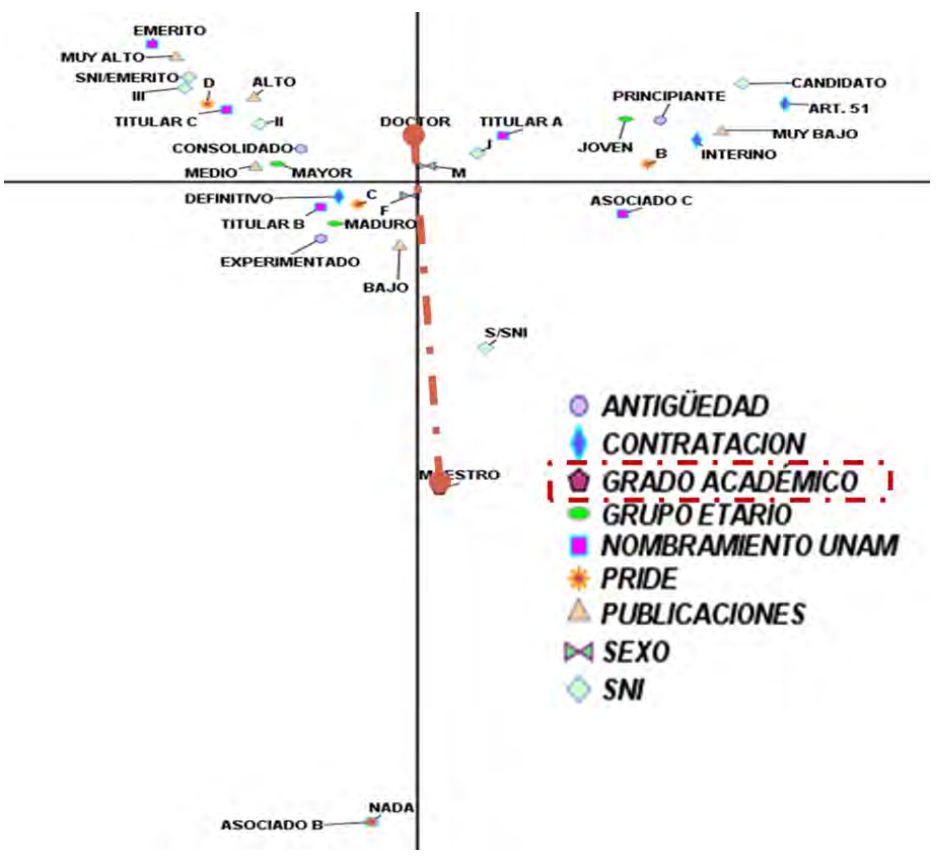

Fuente: Elaboración propia con datos de IISUE, CONACYT, DGAPA, HUMANINDEX, SAEP. 
También se puede observar en un diagrama la lejanía de una de las categorías con respecto a las demás, en el ejemplo del Diagrama 3, se observa que para la variable del SNI, las categorías SNI Emérito y III prácticamente están juntas y el nivel II muy cercano a los anteriores, lo cual indica que los investigadores ubicados en estos niveles del SNI conjugan características muy similares entre ellos, mientras que las categorías que corresponden a los niveles I, candidato e investigadores sin SNI presentan un distanciamiento considerable, en consecuencia hay grandes diferencias entre los perfiles de los investigadores de estos niveles.

Diagrama 3. SNI Emérito

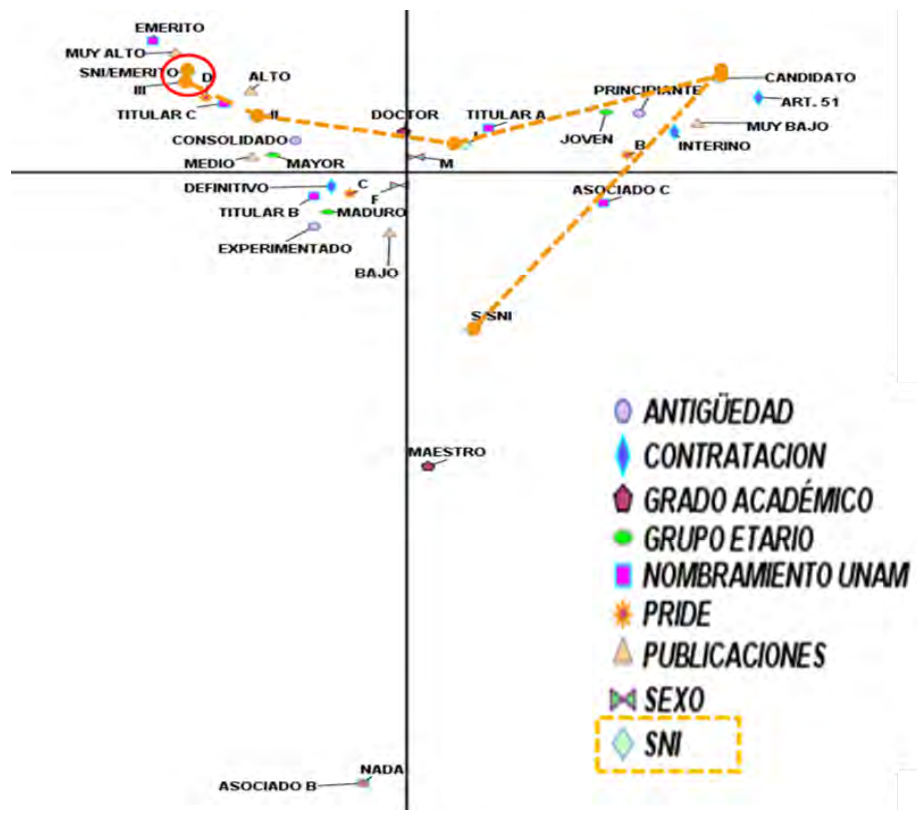

Fuente: Elaboración propia con datos de IISUE, CONACYT, DGAPA, HUMANINDEX, SAEP.

De acuerdo con Bourdieu (1988 y1997) las clases sociales no están dadas, se construyen en el papel, difiere mucho de la visión marxista donde hay un determinismo economicista, en el caso de la propuesta de Bourdieu las clases se construyen a partir de la investigación y la técnica para construir las clases es justamente el ACM. Las clases sociales están definidas por la proximidad entre elementos de diferentes variables, al asociarlas creamos una clasificación, la conjunción de distintas características nos da una clase social particular.

La asociación de variables la llamamos clases sociales, esto es a lo que se refiere Bourdieu cuando plantea que las clases se construyen, lo que vamos haciendo como investigadores es elegir cuál es ese conjunto de puntos que para nosotros conforma un grupo en particular, por la semejanza entre sus características, porque posee recursos materiales y simbólicos (capitales) que lo diferencian de los otros y nos permite explicar cuáles son las prácticas que tienen los sujetos que pertenecen a esa clase. Ya que como menciona Bourdieu (2000, p. 77) "La estructura de las relaciones objetivas entre los agentes determina lo que estos pueden hacer o no hacer". Para el caso específico de esta investigación este acercamiento a la construcción de clases sociales permite identificar en el Diagrama 4, tres clases sociales: Consolidado, Herético y Principiante. 
Diagrama 4. Consolidado, Herético y Principiante.

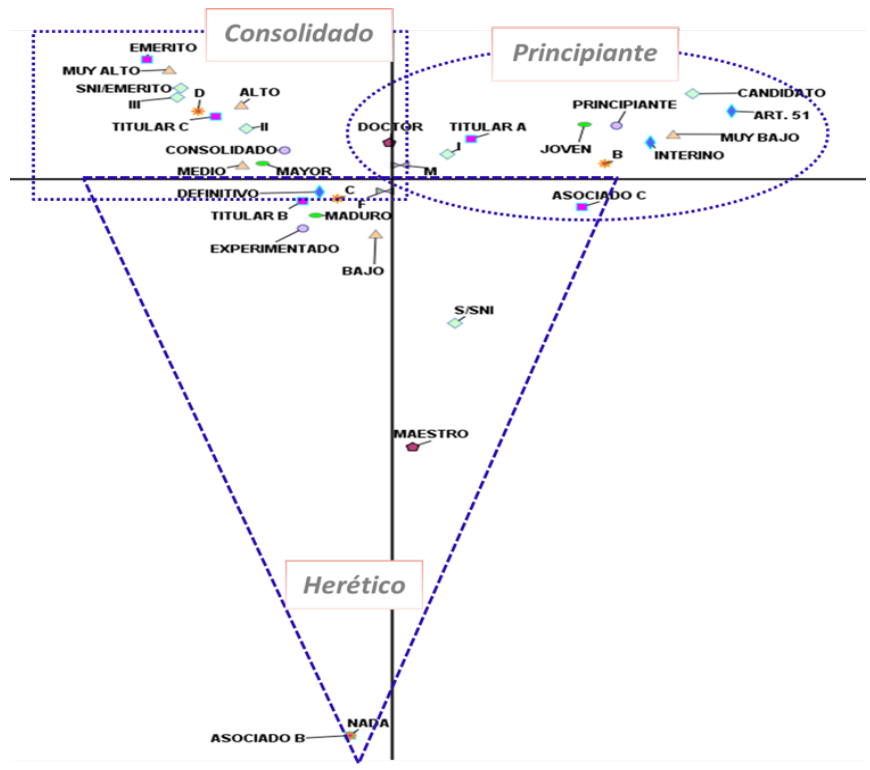

Fuente: Elaboración propia con datos de IISUE, CONACYT, DGAPA, HUMANINDEX, SAEP.

Los diagramas generados por el ACM muestran la estructura de las relaciones objetivas que se establecen en un campo de acuerdo con el volumen y estructura de capitales que poseen los integrantes del mismo, y los agrupamos para formar las clases sociales acordes a la coincidencia, a la cercanía que existe entre ellos y que permite identificarlos como un conjunto que caracteriza a esa clase en particular.

Otra de las ventajas del ACM es que los sujetos están presentes en forma de nubes de puntos, Diagrama 5, no se pulverizan en fórmulas que los convierten en índices, porcentajes, promedios, etc. Las nubes de puntos refieren que hay diferencias entre unos y otros, y la distancia o la proximidad entre ellos nos facilita comprender en qué son diferentes o semejantes a partir de las propiedades que los sujetos poseen y las prácticas que realizan.

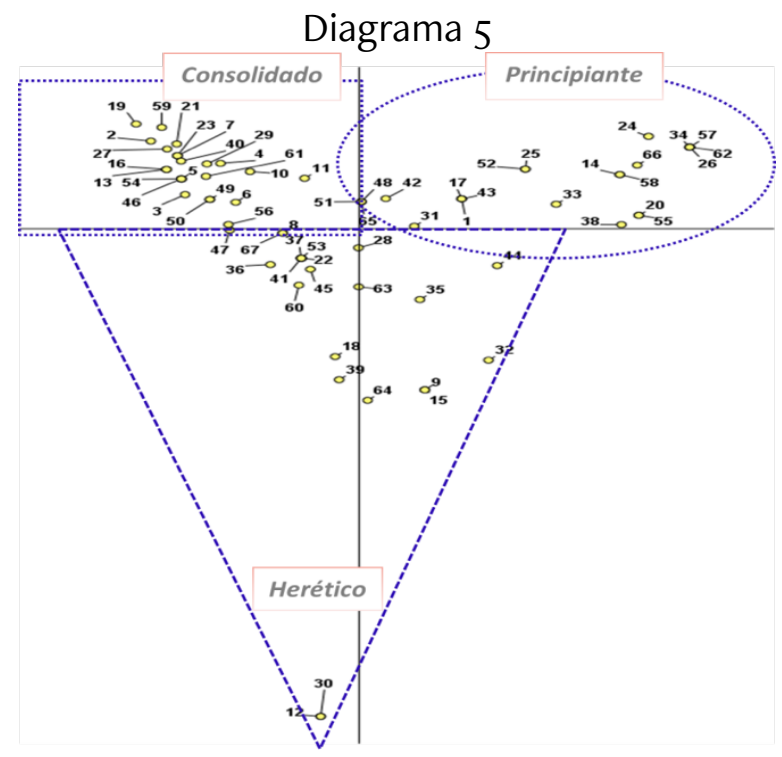

Fuente: Elaboración propia con datos de IISUE, CONACYT, DGAPA, HUMANINDEX, SAEP. 
Este último diagrama permite la visualización de todos y cada uno de los investigadores del IISUE distribuidos en el espacio social, la visualización de la ubicación de cada una de las unidades de análisis, en este caso, la localización de los 67 investigadores del IISUE y agrupados de acuerdo a la clase a la que pertenecen. No obstante, es importante señalar que la distancia entre los miembros de las distintas clases que estructuran el espacio social está dada también y solo en el papel, las relaciones en el espacio físico no suceden así, ahí las personas están próximas, interactúan cotidianamente entre ellas.

Por otro lado, el ACM se constituyó en el método que permitió ubicar a los investigadores del IISUE dentro del espacio social, de acuerdo al volumen y estructura de los bienes que poseen, y seleccionar a 3 investigadores que representen cada una de las clases para la realización de entrevistas, las cuales constituyen un segundo momento metodológico, desde la perspectiva bourdiana, que posibilitará el conocimiento del sentido vivido de las prácticas.

\section{Discusión}

Es importante recordar que las relaciones sociales se configuran de manera desigual en virtud del conjunto de bienes adquiridos por los sujetos que conforman el campo y esos bienes materiales y simbólicos (capitales) intervienen como recursos para poder producir y reproducir la existencia y van creando clases sociales posicionadas en el espacio social, por su posición y relaciones entre las posiciones. En este sentido Bourdieu y Wacquant plantean que,

Cada campo convoca y da vida a una forma específica de interés, una illusio específica, bajo la forma de un reconocimiento tácito del valor de los asuntos en juego y el dominio práctico de sus reglas. Además, este interés específico implícito por la participación en el juego difiere según la posición que se ocupe en él (dominante versus dominado u ortodoxo versus herético) y la trayectoria que condujo a cada participante a su posición (Bourdieu y Wacquant, 2005, p. 175).

Los resultados obtenidos con el ACM permiten interpretar de la siguiente manera los diagramas, ubicamos el en cuadrante superior izquierdo, Diagrama 6, a los investigadores, mujeres y hombres, que poseen los recursos que caracterizan a los investigadores Consolidados, destacados por el reconocimiento obtenido de la comunidad académica, de mayor prestigio y gran conocimiento del oficio, aquí se ubican los mejor provistos de los recursos que exige el campo científico, cuentan con plaza definitiva, con nombramiento de Investigador Emérito y Titular C, nivel SNI Emérito, SNI III y II, participan en el programa de estímulos de la UNAM en la categoría de PRIDE $\mathrm{D}$, tienen grado académico de doctor, respecto a la productividad académica se encuentran en los niveles Muy alto, Alto y Medio, pertenecen al grupo con mayor antigüedad en la práctica de la investigación y también forman parte del grupo etario de mayor edad. 
Diagrama 6. Recursos que caracterizan a los investigadores Consolidados

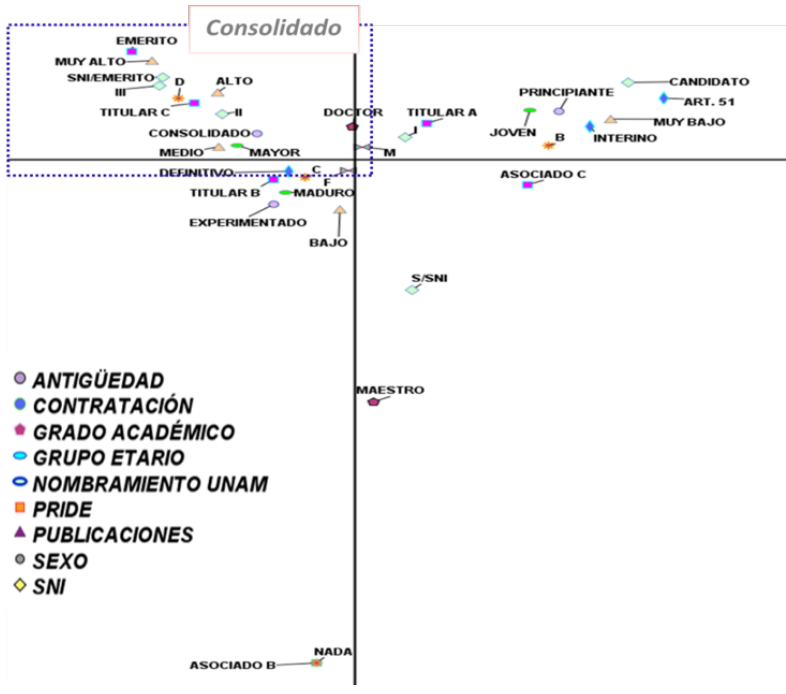

Fuente: Elaboración propia con datos de IISUE, CONACYT, DGAPA, HUMANINDEX, SAEP.

Los investigadores consolidados ocupan la posición dominante, son los investigadores que mejor se han apropiado de los recursos disponibles para destacar en el campo, son los que han incorporado a sus prácticas todas las exigencias de "lo que debe ser un investigador", son quienes durante su trayectoria académica han implementado estrategias específicas para la reconversión de capitales de acuerdo a los momentos históricos que les ha tocado vivir, recordemos que ellos conforman el grupo etario de mayor edad y con más experiencia en la práctica de la investigación, además, por su posición dominante han participado en las luchas por la prescripción del campo de la investigación educativa, les ha tocado definir líneas de investigación, qué temas abordar, crear foros de difusión y debate de las ideas, determinar con quiénes dialogar, precisar dónde publicar, en síntesis crear el ambiente para la vida académica, ya que según Bourdieu "los dominantes son aquellos que llegan a imponer la definición de la ciencia según la cual la realización más acabada de la ciencia consiste en tener, ser y hacer, lo que ellos tienen, son o hacen" (Bourdieu, 1999, p. 82).

Por otro lado, en los dos cuadrantes inferiores se encuentran los investigadores Heréticos, Diagrama 7 , trasgresores del orden social, muestran resistencia a las reglas del campo, ya que por un lado reúnen atributos de conocimiento de la práctica del oficio de la investigación, pertenecen a un grupo experimentado, tienen una amplia trayectoria, conforman el grupo etario de los Adultos con una edad mayor a los 51 años, sin embargo, vistos desde la lógica dominante de los investigadores destacados, se observa cierto rezago con respecto a la carrera académica ya que, aun cuando ocupan plazas definitivas, sus nombramientos son los de menor jerarquía: Titular B, Asociado C o Asociado B, tienen grado académico de maestría, su nivel de productividad académica es Baja, en consecuencia no son parte del SNI y solo algunos participan en el programa de estímulos de la UNAM, PRIDE, en el nivel C. De ahí que, Bourdieu (2008) considera que quienes se ubican en las posiciones marginales tienden a excluir ciertos mecanismos de reproducción, sin romper totalmente con el orden, desvían su trayectoria de una carrera universitaria 'normal'. 
Es importante aclarar que se decidió denominarle Herético al grupo de investigadores que difiere del “tipo ideal” (Weber, 1964) en su carrera académica, con el fin apreciarlos desde otro punto de vista, diferente al de las cualidades de los investigadores destacados, con el fin de indagar a futuro cuáles son los recursos que les han permitido mantenerse dentro del espacio social, cuáles son los capitales que han movilizado para no ser excluidos, cuáles son las estrategias que les han permitido formar parte de los investigadores del IISUE y realizar una trayectoria distinta a los cánones de la carrera académica ascendente y tradicional, diferente a los indicadores de calidad impuestos para la investigación científica y que se centran, principalmente, en una alta productividad académica e integración al SNI, recordemos que la pertenencia al SNI otorga la mayor distinción entre la comunidad científica. Es evidente que los investigadores heréticos no son por supuesto movidos exclusivamente por las fuerzas del campo, estos cuentan con disposiciones, maneras de ser, pensar, y actuar que los pueden llevar a oponerse a la inercia de las fuerzas del campo (Bourdieu, 2000).

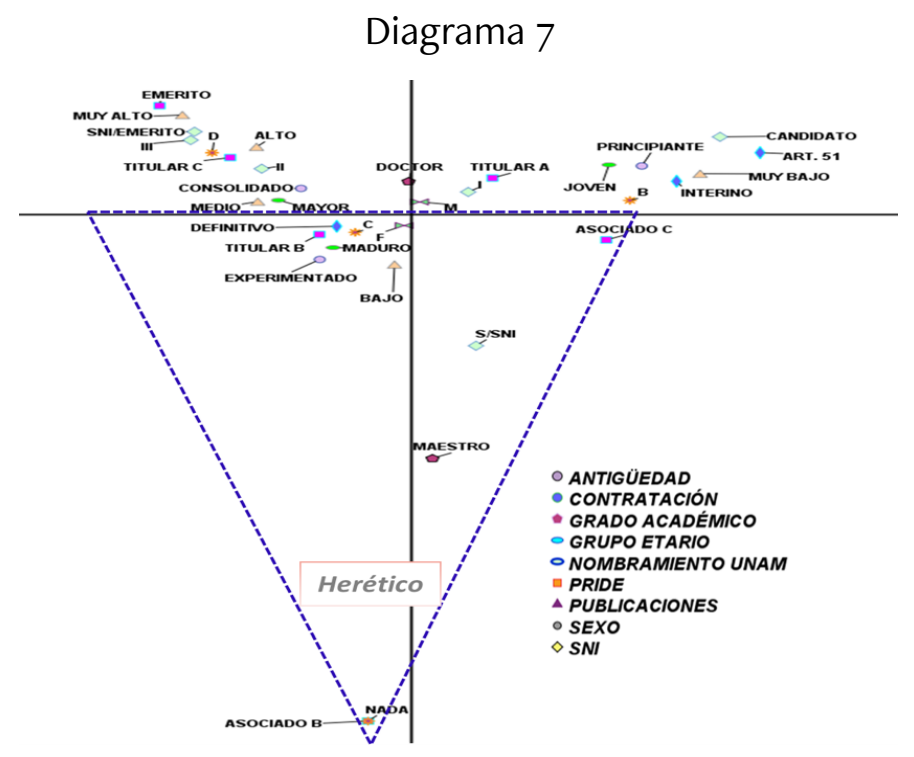

Fuente: Elaboración propia con datos de IISUE, CONACYT, DGAPA, HUMANINDEX, SAEP.

Finalmente, en el cuadrante superior derecho, Diagrama 8, se ubican los investigadores más jóvenes con una edad que oscila entre los 35 y los 50 años, tienen menos de ocho años dentro del Instituto, son Principiantes en el desempeño del oficio, cuentan con una trayectoria corta, sin embargo es importante destacar que a pesar de su corta incorporación al Instituto traen un curriculum robusto, son doctores y tienen las suficientes credenciales como para participar en el programa de estímulos de la UNAM, son PRIDE B y también se han postulado al SNI, y por lo tanto, ya son candidatos e incluso ya han logrado obtener el nivel I, independientemente de que su contratación aun sea de interino o haya sido por artículo 51, es decir, temporal y por obra determinada, y en consecuencia carezcan de estabilidad laboral, por ser el grupo de investigadores que inicia su carrera académica su nivel de productividad es aún Muy bajo.

La institucionalización y profesionalización de la investigación en la UNAM ha pasado por procesos muy trascendentes, ejemplo de ello es la contratación de personal académico con nombramiento de investigadores de tiempo completo; con el propósito de realizar una selección académica más rigurosa del personal de nuevo ingreso, por la vía de los hechos, es decir sin 
necesidad de modificar la norma, se ha establecido como nivel inicial de la carrera de investigador la contratación de personal con plazas de asociado C (Soberón, 1995), y el EPA considera como requisitos para esta categoría: tener grado de maestría, haber trabajado cuando menos tres años en labores de docencia e investigación y publicar trabajos que acrediten su competencia; al adicionarle opciones a este último requisito como: o ser doctor, o dirigir seminarios y tesis o impartir cursos de manera sobresaliente, se elevan los perfiles de ingreso y en consecuencia se contrata a aquellos aspirantes con un curriculum destacado.

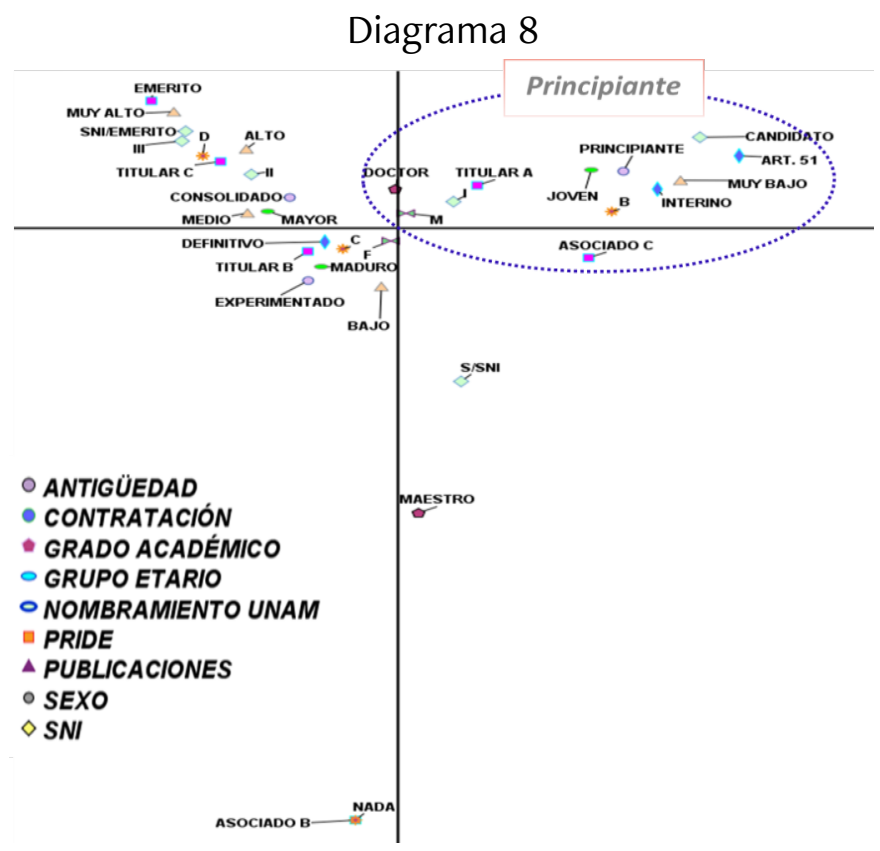

Fuente: Elaboración propia con datos de IISUE, CONACYT, DGAPA, HUMANINDEX, SAEP.

Para los Principiantes la ventaja social de haberse constituido en investigadores en la época actual, consiste en que hay normas precisas para la investigación científica de su campo, hay tendencias, hay regularidades, hay tradiciones claras (Díaz-Barriga, 2014), y éstos las han incorporado tan bien que se presentan a la competencia por las plazas cumpliendo sobremanera los requisitos, dado que cada vez se presentan al proceso de selección con las mejores credenciales posibles, y ello les ha permitido postularse casi inmediatamente al SNI, por ejemplo. Así mismo, el SNI al normar las prácticas científicas y otorgar reconocimiento a las mismas está estructurando el campo y los tipos de capitales que se ponen en juego para pertenecer al mismo, el SNI se constituye, de este modo, en el principal elemento de diferenciación y clasificación de la comunidad científica.

\section{Conclusiones}

Diversos trabajos referentes a la formación de investigadores educativos versan sobre una polarización en los procesos formativos, que van desde aquellas de carácter reflexivo y "profesionalizante" hasta aquellas de corte investigativo (Acuña y Pons, 2019), de igual manera, se presentan aquellos sobre la configuración y evolución del campo de la investigación educativa (Colina, 2011; Colina y Osorio, 2004; Latapí, 1994; Ramírez y Weiss, 2004), así como los referidos a la producción de conocimiento en investigación educativa (Gutiérrez, 2006), sin embargo, existe un denominador común, sustentan el objeto de estudio desde perspectivas metodológicas focalizadas en la descripción presente o histórica, diferenciándose con este estudio por 
estar centrado en la construcción y articulación de las condiciones objetivas de los agentes y su correspondiente práctica de la investigación educativa mediante un análisis estadístico, recuperando la historia hecha institución y la historia hecha cuerpo.

Así mismo, en esta investigación se recuperó un posicionamiento teórico-metodológico de corte relacional, no solamente para analizar las prácticas investigativas de los agentes con base en su posición dentro del espacio social, sino también, para la construcción de clases sociales a partir de las trayectorias y de su volumen y estructura de capital. En consecuencia, la posesión de bienes materiales y simbólicos (capitales) puesta en juego por los investigadores para posicionarse en el espacio social (campo) constituyen, a la vez, las estrategias de reproducción de su clase, reproduciendo los mecanismos de poder y desigualdad que sustentan la estructura, ya que de esta manera se perpetúa la sociedad en su conjunto. Recordando que toda práctica es una estrategia, producida por un sujeto que actúa independientemente de su conciencia y voluntad, pero que sus prácticas son resultado de la relación dialéctica entre las estructuras objetivas externas y las estructuras objetivas internas, incorporadas bajo la forma de disposiciones.

De tal modo que, conocer la estructura de las posiciones y la relación entre posiciones de los investigadores del IISUE, a través del análisis de correspondencia múltiples, ha permitido darle solidez metodológica a la investigación, presentar de una manera rigurosa el manejo de datos estadísticos que nos arroja diagramas que fotografían en un momento determinado de la historia a los sujetos de la investigación; para Bourdieu el análisis estadístico "es para el sociólogo lo que la experimentación es para el físico" (Bourdieu et al., 1963, p. 10), en este sentido, atrevernos a emplear métodos matemáticos para comprender una realidad social, más allá de sus representaciones e imaginarios, ha sido hacer funcionar una parte de la propuesta teórico-metodológica de Pierre Bourdieu.

Por otra parte y para continuar y complementar esta investigación, el ACM será también la herramienta que permite comprender "desde dónde habla" un investigador, por consiguiente, referirnos a la posición que ocupa en el campo y a partir de la cual emite su punto de vista sobre sus prácticas de investigación y del campo disciplinar en su conjunto; Bourdieu señala que "Los agentes sociales están insertados en la estructura, en posiciones que dependen de su capital y desarrollan estrategias que, en sí mismas, dependen en gran parte de esas posiciones, en los límites de sus disposiciones" (Bourdieu, 2000, p. 82). Por lo cual, recuperar el sentido vivido de las prácticas sociales a partir de la posición ocupada en el campo será el segundo momento metodológico que Bourdieu nos plantea para comprender la realidad social, y es en consecuencia la estrategia metodológica que sigue a este estudio y queda como tarea pendiente.

Si bien las consideraciones finales aquí presentadas proveen una aproximación para comprender el espacio social del IISSUE, es importante mencionar que éstas pueden no ser totalizadoras toda vez que, tal como lo refiere Guerrero, es importante considerar "la yuxtaposición de los campos en un mismo espacio social; esta consideración implica que aquello que para un campo determinado (...) puede considerarse como una disposición, para otro campo (...) puede considerarse como un habitus y viceversa" (2020, p. 130). De acuerdo con lo anterior, una recomendación para estudios posteriores radica en considerar que los agentes sociales se desempeñan en diferentes campos de manera simultánea, por lo que las disposiciones o habitus pueden presentar efectos de alodoxia que es importante estudiar. 


\section{Referencias}

Acuña, L., \& Pons, L. (2019). Itinerarios de la formación de investigadores educativos en México. REICE. Revista Iberoamericana sobre Calidad, Eficacia y Cambio en Educación, 17(4), 27-57. https://doi. org/10.15366/reice2019.17.4.002

Baranger, D. (2009). Construcción y análisis de datos. Introducción al uso de técnicas cuantitativas en la investigación social. Posadas.

Bourdieu, P. (1988). Cosas dichas. Gedisa.

Bourdieu, P. (1990). Espacio social y génesis de las clases. En P. Bourdieu. Sociología y Cultura, (pp. 281309). Grijalbo-CNCA.

Bourdieu, P. (1997). Razones prácticas. Anagrama.

Bourdieu, P. (1999). Intelectuales, política y poder. Eudeba.

Bourdieu, P. (2000). Los usos sociales de la ciencia. Nueva Visión.

Bourdieu, P. (2001a). Poder, derecho y clases sociales. Desclée de Brouwer.

Bourdieu, P. (2001b). Describir y prescribir: las condiciones de posibilidad y los límites de la eficacia política. En, P. Bourdieu. ¿Qué significa hablar? Economía de los cambios lingüísticos, (pp. 96-104). Akal.

Bourdieu, P. (2011). Las estrategias de la reproducción social. Siglo XXI.

Bourdieu, P. (2014). Sobre el Estado. Cursos en el Collège de France (1989-1992). Anagrama.

Bourdieu, P., Darbel, A., Rivet, J. P., \& Seibel, C. (1963). Travail et travailleurs en Algérie. Mouton.

Bourdieu, P., \& Wacquant, L. (1995). Respuestas por una antropología reflexiva. Grijalbo.

Bourdieu, P., \& Wacquant, L. (2005). Una invitación a la sociología reflexiva. Siglo XXI.

Cabrera, D. M. (2013). La investigación educativa en tres revistas académicas mexicanas 19922002. Asignaciones de Sentido. Perfiles Educativos, 35(142), 108-125. https://doi.org/10.22201/ iisue.24486167e.2013.142.42578

Colina,A. (2011). El crecimiento del campo de la investigación educativa en México. Un análisis a través de sus agentes. Perfiles Educativos, 33(132), 10-28. https://doi.org/10.22201/iisue.24486167e.2011.132.24894

Colina, A. y Osorio, R. (2004). Los agentes de la investigación educativa en México. Capitales y habitus. CESUUNAM/Plaza y Valdés.

Chehaibar, L. M. \& Paniagua, E. (2009). XXX Aniversario de Perfiles Educativos. Perfiles Educativos, 31(123), 110-117. https://doi.org/10.22201/iisue.24486167e.2009.123.18818

CONACyT. (2017, octubre 3). Sistema Nacional de Investigadores. https://conacyt.mx/sistema-nacionalde-investigadores/

Díaz-Barriga, Á. (2014, junio 17-20). Tradiciones académico-disciplinares en educación en México [Conferencia]. Congreso Internacional Epistemologías y metodologías de la investigación en educación, México. http://www.iisue.unam.mx/afirse/programa.php.

Guerrero, L. (2020). Capitales, habitus y disposiciones de profesores universitarios. Una aproximación a partir de sus trayectorias académicas. Religación. Revista de Ciencias Sociales y Humanidades, 5(25), 117-131. https://doi.org/10.46652/rgn.v5i25.672

Gutiérrez, G. (2006). Comunidades especializadas en investigación educativa en México. Cultura y representaciones sociales, 1(1), 163-176. https://bit.ly/3pgk8zr

Gutiérrez, G. (1998). Orígenes de la institucionalización de la investigación educativa en México. Revista Mexicana de Investigación Educativa, 3(5), 13-38. https://www.comie.org.mx/revista/v2018/rmie/index. php/nrmie/article/view/1031

Latapí, P. (1994). La investigación educativa en México. Fondo de Cultura Económica. 
Moscoloni, N. (2011). Las nubes de datos: métodos para analizar la complejidad. Universidad Nacional de Rosario.

Ramírez, R. \& Weiss, E. (2004). Los investigadores educativos en México: Una aproximación. Revista Mexicana de Investigación Educativa, 9(21), 501-514. http://www.comie.org.mx/revista/v2018/rmie/ index.php/nrmie/article/view/857

Soberón, G. (1995). Cincuenta años de la Coordinación de la Investigación Científica y del Consejo Técnico de la investigación científica. Mimeo, UNAM.

UNAM. (s/f). Estatuto del Personal Académico. https://cutt.ly/oYRaYVX

UNAM/CISE. (1983). El Programa de trabajo del Centro de Investigaciones y Servicios de la UNAM (CISE). Estudios sociológicos, 1(3), 641-644. https://doi.org/10.24201/es.1983v1n3.1335

UNAM/CESU. (1997). Encuentro académico. XX aniversario del CESU.

UNAM/CESU. (2000). Memoria UNAM 2000, CESU. https://cutt.ly/IYRaBZA

UNAM/CESU. (2006). Propuesta del Centro de Estudios sobre la Universidad para convertirse en instituto. Perfiles Educativos, 28(114), 169-189.

UNAM/HUMANINDEX. (2019). Base de datos bibliográfica de humanidades y ciencias sociales. UNAM/ HUMANINDEX. https://bit.ly/3EaFQLJ

UNAM/IISUE. (2016). Instituto de Investigaciones sobre la Universidad y la Educación. UNAM/IISUE. https://www.iisue.unam.mx

UNAM/IISUE. (2018). Plan de desarrollo institucional del Instituto de Investigaciones sobre la Universidad y la Educación de la UNAM 2018-2022. UNAM/IISUE. https://bit.ly/3D7UjGS

UNAM/SAEP. (2017). Sistema Integral de Administración Escolar del Posgrado (SIAE-P). UNAM/SAEP https://www.saep.unam.mx/v2/inscripcion/insc5.php?enlace=FILOSo10.

Weber, M. (1964). Economía y sociedad: esbozo de sociología comprensiva. Fondo de Cultura Económica.

\section{AUTORES}

Sara Bravo Villanueva. Socióloga, maestra en pedagogía, actualmente estudiante del doctorado en pedagogía en la Facultad de Filosofía y Letras de la Universidad Nacional Autónoma de México (UNAM).

Luis Arturo Guerrero Azpeitia. Doctor en Ciencias de la Educación por la Universidad Autónoma del Estado de Hidalgo (UAEH, México), Maestro en Ciencias con Orientación en Enseñanza de las Matemáticas, Ingeniero en Electricidad. Actualmente es profesor-investigador en la Universidad Politécnica Metropolitana de Hidalgo. 